\title{
Highly Efficient Three Wavelength WOLEDs by Controlling of Electron-Transfer
}

\author{
Ho-Cheol Park, ${ }^{+\neq}$Jong-Wook Parks ${ }^{\$, *}$ and Seong-Geun $\mathrm{Oh}^{\dagger}$ \\ 'Department of Chemical Engineering, Hanvang Lniversity, Seoul 133-791, Korea \\ Fistorm co. Ltd. Sungnam +63-760. Korea \\ "Department of Chemistry Display Research Center, The Catholic Lniversity of Korea, \\ Pucheon +20-743. Korea. ${ }^{*}$ E-mail: hahapankiâcatholic ac. kr \\ Received Jine 3, 2009. Accepted Altgust 20, 2009
}

\begin{abstract}
By controlling the number of electrons transferred to the emitting laver, highly efficient three-wavelength WOLEDs were fabricated. Such WOLEDs are different from those made using simple stacking of $\mathrm{RGB}$ emitting layers in that the movement distribution of electrons transfered to enitting layer could be adjusted using the difference in LUMO energy level and that lights of all 3 wavelengths could be emitted through appropriate arrangement of RGB emitting layers. WOLED device with the structure of m-MTDTA (40 nm)/NPB (10 nm)/ Coumarin6 doped Alq3 $(3 \%)(8 \mathrm{~mm}) /$ Rubrene doped NPB (5\%) (15 nm)/NPB (2 nn)/DPVBi (20 nm)/Alq $(20 \mathrm{~nm}) / \mathrm{LiF}$ (1 nm)/Al (200 nm) showed high luminance efficiency of $8.9 \mathrm{~cd} / \mathrm{A}$ and color purity of $(0.31,0.40)$. In addition. WOLED device with the thickness of non-doped NPB layer increased from $2 \mathrm{~nm}$ to $3 \mathrm{~nm}$ to increase blue light emission showed a luminance efficiency of $7.6 \mathrm{~cd} / \mathrm{A}$ and color purity of $(0.28,0.36)$.
\end{abstract}

Key Words: Three-wavelength WOLED. Electron-transfer. Non-doped NPB

\section{Introduction}

After the first commercialization in 1993. Organic Light Enitting Diodes (OLEDs) are expanding their application to fields that had been occupied by existing displaỵs such as mobile information devices. mobile phones and mp3 players and are recently receiving attention as the most prominent candidate of new solid-state lighting and large surface flat panel display. ${ }^{1-4}$ OLED for solid-state lighting can be minufactured using a plate with comparatively low electric power consumption. Since it can be made flexible and does not contain emvironmental contaminants such as heavy metals. many studies are being conducted on OLED for next generation light source technology. ${ }^{5}$ Method of embodiment of WOLED include multilayer structure that embodies three primary colors or complementary colors." structure in which multiple dopants are doped into a single layer. and stnucture that induces white light by creating an exciplex. Among these methods, multilayer structure is being widely used because it is comparatively easy to manufacture and allows selection of light to be emitted.

Different from single colored OLED. color purity of WOLEDs is difficult to be adjusted because WOLEDs are composed of two or more emitting layers. Especially. although multilay̧ered WOLEDs can conceptually provide stable efficiency. white color can only be achieved when the recombination region of hole and electron is appropriately distributed within the three emitting inside layers. Also. in order to change the color purity of white color doping concentration of emitting layer has to be varied or emitting material used must be changed. WOLED can also be made using organic layers such as hole blocking layer to adjust color purity of white light or to obtain three colors. Devices in which such additional carrier control layer is introduced show high driving voltage and low efficiency characteristics. ${ }^{9.10}$
Accordingly, this paper demonstrated new RGB three-wavelength WOLEDs that differ from existing WOLEDs in that color purity can easily be adjusted and high efficiency can be achieved. Such WOLEDs contain organic layers that control the movement of electrons, and they were composed of appropriate distribution of RGB enutting layers with consideration on energy level so that all three wavelengths could be emitted.

\section{Experimental}

In order to smoothen hole injection $4.4^{\prime} \cdot 4^{\prime \prime}$-tris ( $N-3-$-methylphenyl-k-phenyl-amino)-triphenylamine ( $m$-MTDATA) with similar HOMO level as ITO was used as a hole injection layer.

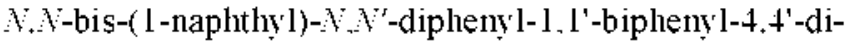
amine (NPB) was used as a hole transport layer. 5,6.11,12-tetrapheny ltetracene (Rubrene) was doped to NPB with 5 wt\% ratio to be used as a red light emitting layer. 3-(Benzothiazol-2-yl)-7(dimethylanino) chroman-2-one (Coumarin6, C6) was doped at $3 \mathrm{wt} \%$ ratio to Tris-(8-hydroxyquinoline) aluminium ( $\mathrm{Alq}_{3}$ ) in order to be used as a green light emitting layer and 4,4 '-bis(2,2'-diphenyl vinyl)-1, 1'-biphenyl (DPVBi) was used as a blue light emitting layer. $\mathrm{Al}_{\mathrm{q}_{3}}$ and $\mathrm{LiF} / \mathrm{Al}$ were respectively used as an electron transport layer and cathode. All organic materials used in this experimental were purchased for OLED device from Sensient Imaging Technologies $\mathrm{GmbH}$.

ITO substrate used has luminance area of $2 \mathrm{~mm} \times 3 \mathrm{~mm}$ and sheet resistance of $10 \Omega$ /square. ITO substrate was used for production of devices after cleaning the surface using organic solvents and DI water and then performing pretreatment with $\mathrm{O}_{2}$ plasna. For vapor deposition of all organic natters and cathode. degree of vacuum within the chamber maintained a pressure under $1 \times 10^{-3}$ torr. The deposition rate was controlled by varying the heating current supplied to evaporation source and moni- 


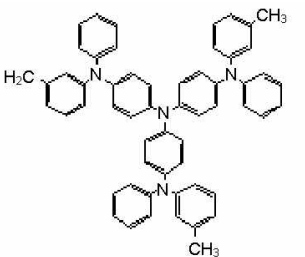

m-MTDATA

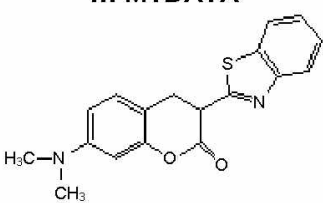

Coumarin6
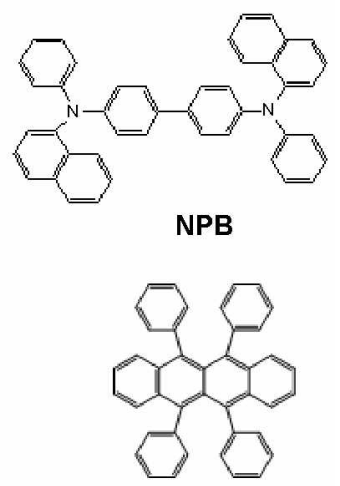

Rubrene

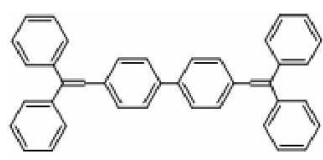

DPVBi

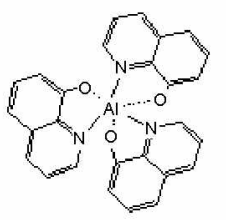

$\mathrm{Alq}_{3}$

\begin{tabular}{|c|}
\hline Alq $20 \mathrm{~nm}$ \\
\hline DPVBi $20 \mathrm{~nm}$ \\
\hline C6 $(3 \%)$ doped Alq $8 \mathrm{~nm}$ \\
\hline Ru $(5 \%)$ doped $\mathrm{NPB} 15 \mathrm{~nm}$ \\
\hline NPB $10 \mathrm{~nm}$ \\
\hline m-MTDATA $40 \mathrm{~nm}$ \\
\hline
\end{tabular}

Device A

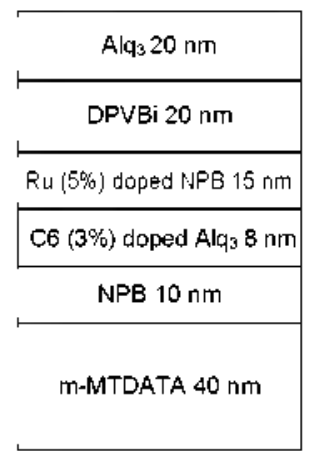

Device B

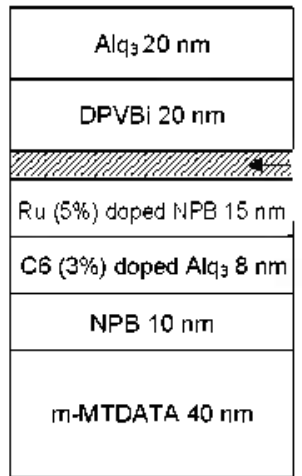

Device $\mathrm{C}$

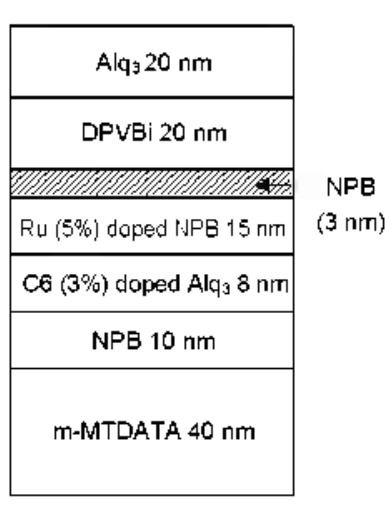

Device $\mathrm{D}$

Figure 1. Chemical Structures of organic materials used and layer configuration of WOLEDs

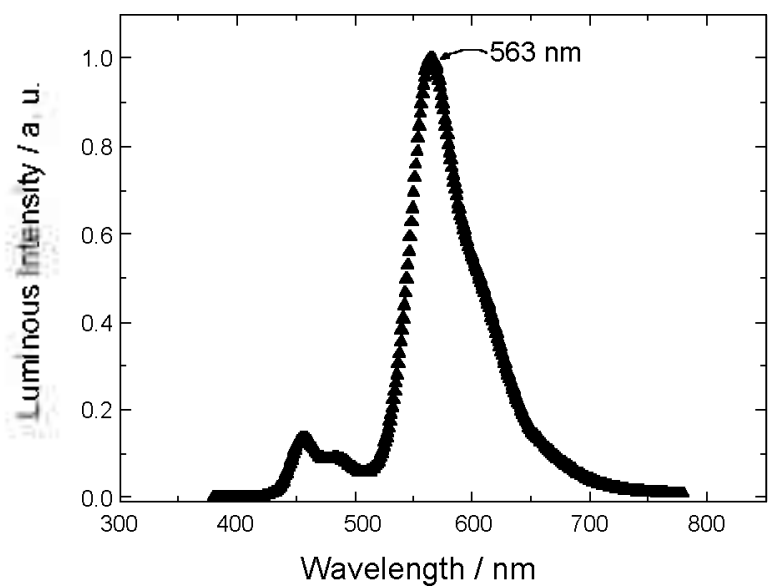

Figure 2. EL spectrum of Device A. Device A: ITO/m-MTDATA 40 min/NPB 10 m $1111 /$ Rubrene ( $5 \%$ ) doped NPB $15 \mathrm{~mm} / \mathrm{C} 6$ (3\%) doped Alq $8 \mathrm{~nm} / \mathrm{DPVBi} 20 \mathrm{~nm} / \mathrm{Alq}_{3} 20 \mathrm{~nm} / \mathrm{LiF} 1 \mathrm{~nm} / \mathrm{Al} 200 \mathrm{~nm}$. tored in situ by a quartz cry stal sensor. which could provide very reproducible experimental result.

\section{Result and Discussion}

Figure I shows the device configuration of WOLEDs and the chemical structures of organic materials used for this experiment. Device A is a WOLED composed simply of sequential stacking of RGB emitting layers. Figure 2 is the EL spectrum result of Device A. In case of Device A with a simple sequential layer structure. most of the injected holes and electrons moved to rubrene with lowest energy level and emitted light by reconbining mainly at the rubrene layer. showing not so good white color purity of $(0.460 .0 .470)$ (see Table 1$)$.

Device $B$ is a stnucture for green light emission in which rubrene doped NPB layer was positioned behind $\mathrm{Alq}_{3}$ layer doped with coumarin6 to arrange LUMO energy level of red-emitting nubrene in the form of a well within the device. As shown in

Table 1. EL perfornances of Device A - D at $10 \mathrm{~mA} / \mathrm{cm}^{2}$.

\begin{tabular}{ccccccc}
\hline Device namne & Voltage $(\mathrm{V})$ & Luminance $\left(\mathrm{cd}^{\prime} / \mathrm{m}^{2}\right)$ & CIE coordinates & EL Peak $(\mathrm{nm})$ & $\mathrm{cd} / \mathrm{A}$ & $\mathrm{lm} / \mathrm{W}^{\prime}$ \\
\hline $\mathrm{A}$ & 5.9 & 763 & $\mathrm{x}=0.460, \mathrm{y}=0.473$ & 564 & 7.6 & 4.0 \\
$\mathrm{~B}$ & 6.7 & 1459 & $\mathrm{x}=0.372, \mathrm{y}=0.543$ & 557 & 14.6 & 6.8 \\
$\mathrm{C}$ & 6.9 & 887 & $\mathrm{x}=0.310, \mathrm{y}=0.406$ & 455 & 8.9 & 4.0 \\
$\mathrm{D}$ & 7.0 & 760 & $\mathrm{x}=0.280, \mathrm{y}=0.360$ & 455 & 7.6 & 3.4 \\
\hline
\end{tabular}

"Power efficiencies are calculated from ed A with the assumption of a lambertian emission. 


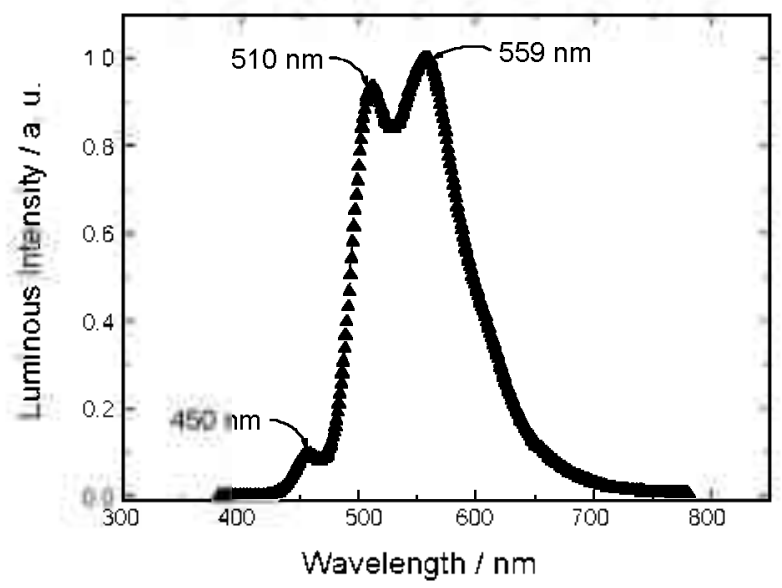

Figure 3. EL spectrum of Device B. Device B: ITO/m-MTDATA 40 $\mathrm{nm} / \mathrm{NPB} 10 \mathrm{~nm} / \mathrm{C} 6(3 \%)$ doped Alq; $8 \mathrm{~nm} /$ Rubrene $(5 \%)$ doped NPB $15 \mathrm{~mm} / \mathrm{DPVBi} 20 \mathrm{~mm} / \mathrm{Alq} ; 20 \mathrm{~mm} / \mathrm{LiF}$ l $\mathrm{mm} / \mathrm{Al} 200 \mathrm{~mm}$

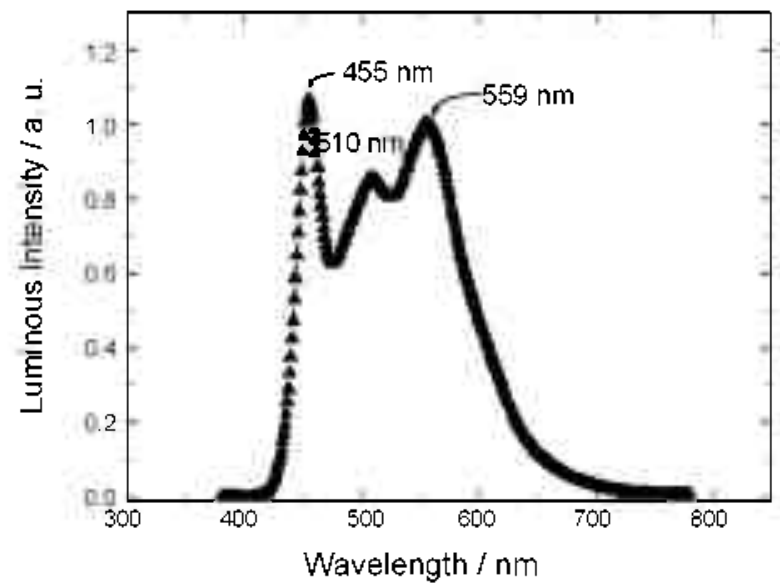

Figure 4. EL spectrum of Device C. Device C: ITO/n1-MTDATA 40 $\mathrm{nm} / \mathrm{NPB} 10 \mathrm{~mm} / \mathrm{C} 6$ (3\%) doped Alq $8 \mathrm{~nm} /$ Rubrene ( $5 \%$ ) doped NPB $15 \mathrm{~nm} / \mathrm{NPB} 2 \mathrm{~nm} / \mathrm{DPVBi} 20 \mathrm{~nm} / \mathrm{Alq}_{3} 20 \mathrm{~nm} / \mathrm{LiF} 1 \mathrm{~nm} / \mathrm{Al} 200 \mathrm{~nm}$.

Figure 3, green light luminance of $\mathrm{Alq}_{3}$ doped with coumarin6 was found to have largely increased by changing the position of NPB layer doped with rubrene. However. color purity of WOLED was still low at $(0.372 .0 .543)$ because of weak blue light emission (Table 1).

The configuration sequence of ITO/Blue/Green/Red/Cathode device is interesting point. however in here we mainly focus on the additional layer system for white OLED. Accordingly, non-doped NPB layer with $2 \mathrm{~nm}$ thickness was additionally introduced next to the red light emitting layer as in Device $\mathrm{C}$ to increase blue light emission. NPB used as a hole transport layer and red color host can limit the movement of electrons due to the high $\mathrm{LUMO}$ value of $2.4 \mathrm{eV}$. Figure 4 shows the EL spectnum of Device C. By introducing a $2 \mathrm{~nm}$ NPB layer. blue light emission largely increased to show a luminance efficiency of $8.9 \mathrm{~cd} / \mathrm{A}$ and color purity of $(0.310 .0 .406)$ (Table 1$)$. When the current density was increased to $50 \mathrm{~mA}^{-\mathrm{cm}^{2}}$ in Device $\mathrm{C}$, luminance efficiency was increased to about $11 \mathrm{~cd} / \mathrm{A}$ and $\mathrm{CIE}$ value was $(0.33,0.45)$. In case of Device $D$ in which the thickness of non-doped NPB was increased to $3 \mathrm{~nm}$, as shown in

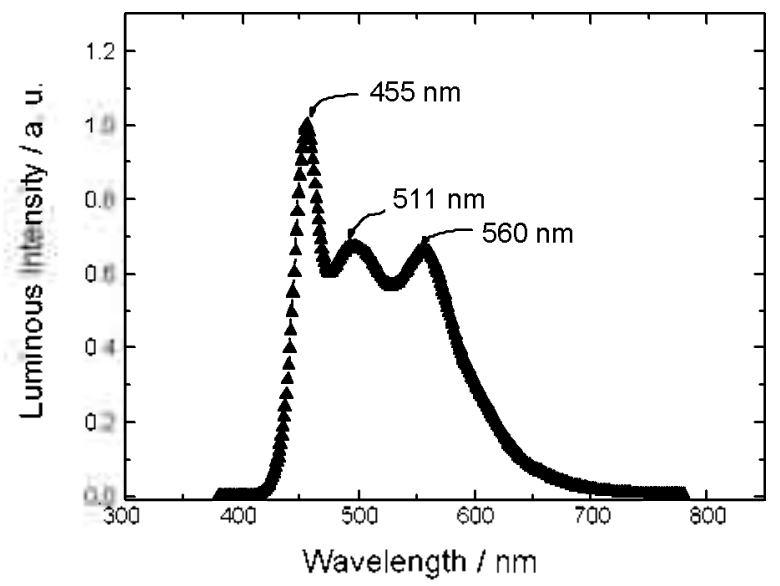

Figure 5. EL spectrum of Device D. Device D: ITO/m-MTDATA 40 $\mathrm{nm} / \mathrm{NPB} 1 \mathrm{cmm} / \mathrm{C} 6(3 \%)$ doped Alqq $8 \mathrm{~nm} / \mathrm{Rubrene}(5 \%)$ doped NPB $15 \mathrm{~mm} / \mathrm{NPB} 3 \mathrm{~nm} / \mathrm{DPVBi} 20 \mathrm{~mm} / \mathrm{Alq}$ : $20 \mathrm{~nm} / \mathrm{LiF} \mathrm{lmm} / \mathrm{Al} 200 \mathrm{~nm}$.

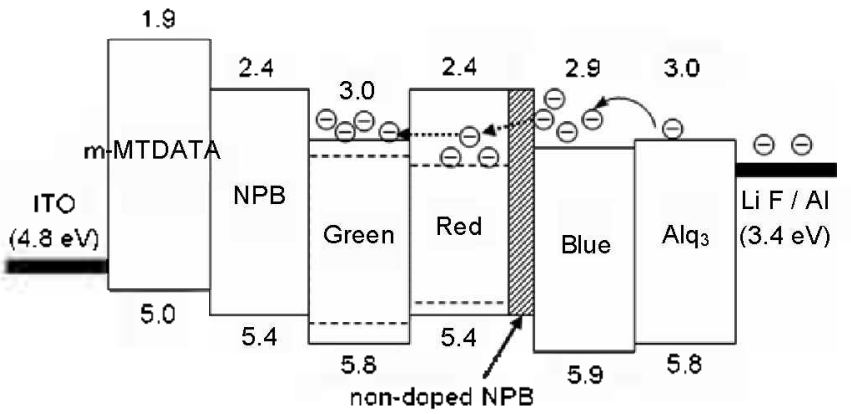

Figure 6. Energy level structure of WOLED device.

Figure 5, blue light emission became even stronger and device shows luminance efficiency of $7.6 \mathrm{~cd} / \mathrm{A}$ and color purity of $(0.280,0.360)$. The balanced white light performance through relatively stronger blue light was mainly controlled by the thickness of non-doped NPB layer. not doping concentration. Luminescence perfornnances of Device A-D are summarized in Table 1

NPB broadly used as a hole transport layer was verified through TOF experiment to have lower electron mobility in comparison to hole mobility. ${ }^{11.12}$ In addition, electrons cannot easily move through NPB because NPB has a relatively high LUMO value of $2.4 \mathrm{eV}$. In this perspective. if NPB is used simply for the use other than hole transport layer. movement barrier on electrons will be created and then the device will show the increased driving voltage and low efficiency. However. as shown in the results of devices summarized in Table 1. driving voltage of Device $C$ was only $1.0 \mathrm{~V}$ higher than the voltage of Device A under current density of $10 \mathrm{~mA} / \mathrm{cm}^{2}$ and showed higher luminance efficiency of $8.9 \mathrm{~cd} / \mathrm{A}$. As a result. power efficiency was maintained at the same level while an improved color coordinate of white light could be obtained.

In the emitting layer. energy transfer between host and dopant can be done in two ways, one in which injected electric charge is transferred to the dopant by passing through the host and the other in which charge can directly be transferred to the dopant without passing through the host. ${ }^{13.14}$ Therefore, explaining the 
process of electron movement in Device C using the energy level figure shown in Figure 6 . electrons injected by the cathode first pass through the blue DPVBi layer and encounter LUMO energy barrier of NPB. At this moment. some of electrons pass through non-doped NPB layer with $2 \mathrm{~nm}$ thickness and the rest cannot. Electrons passing through non-doped NPB layer can move on to the nibrene LUMO energy level within NPB layer doped with rubrene and sequentially to $\mathrm{Alq}_{3}$ layer doped with coumarin6. On the other hand. electrons that fail to pass through non-doped NPB can only emit light by recombining with holes at the DPVBi layer. Ultimately, electrons can be used evenly in RGB emission by moving around without large energy barrier. If thickness of non-doped NPB layer is increased. the number of electrons passing through will be decreased and light emission of DPVBi will become even stronger. Looking at the EL spectrum result of Device D shown in Figure 5. the increase in luminance flux of blue emission was observed from the increase in thickness of non-doped NPB layer to $3 \mathrm{~nm}$. Accordingly, by using doped NPB as emitting layer and thin non-doped NPB layers with thickness of $2 \sim 3 \mathrm{~nm}$, highly efficient three-wavelength WOLED that can control the movement of electrons could be produced. Further studies on various current density including I-V-L result as well as the device property with highly efficient emitting materials such as phosphorescence are underway.

\section{Conclusion}

Using LUMO energy level difference in organic layers. movement of electrons injected to emitting layers was controlled. Highly efficient WOLEDs were made through appropriate arrangement of RGB emitting layers. Such WOLEDs used rubrene doped NPB layer as red light emitting layer and non-doped NPB layer with thickness of $2-3 \mathrm{~nm}$ to control the movement of electrons. This WOLED device showed high luminance efficiency of $8.9 \mathrm{~cd} / \mathrm{A}$ and color purity of $(0.31,0.40)$. WOLED derice with 3 nm non-doped NPB layer showed luminance efficiency of 7.6 $\mathrm{cd} / \mathrm{A}$ and color purity of $(0.28,0.36)$. Therefore. highly efficient three wavelength WOLED was achieved by introducing electron balancing layer. It could be applicable to various emitting sequence inside of OLED as well as the various doped-material system.

Aclonowledgments. This research was supported by a grant (Catholic Univ:) from the Fundamental R\&D Program for Core Technology of Materials funded by the Ministry of Knowledge Economy (MKE), Republic of Korea. This work was supported by the National Research Foundation of Korea (NRF) grant funded by the Korea government (No. M2009A010800007).

\section{Refeience}

1. Baldo, M. A.; Thompson, M. E.: Fonest, S. R. Namme 2000, 403 , 750 .

2. Wu, C. C.: Lin, Y. T.; Wong, K. T.: Chen, R. T.; Chien, Y. Y. Ad Nater 2004, 16,61.

3. Bum, P. L.; Lo, S. C. Samuel, I. D. W. Adw: Mater: 2007, 19, 1675.

4. Wu, K. C.: Ku, P. J.: Lin, C. S.; Shih, H. T.; Wu, F. I.: Huang, M. T.: Lin, J. T.: Chen, I. C.; Cheng, C. H. Adr. Funct. A later 2008, $18,67$.

5. (a) Pounds, T. T.; Wilsoon, R. T.; Grizzi, I. SID 2007 Digest, 875 ; (b) Yeh, P. C.: Hwang, S. W.: Ma, J. W.; Ho, M. H.; Hsu, S. F; Chen, C. H. SHD 2007 Digest, 871 .

6. (a) D'Andrade, B. W: Thomson, M. E; Forrest, S. R. Adr. Water: 2002, 14, 147: (b) Kido, I.: Kimura, M.: Nagai, K. Science 1995. 267,1332 .

7. Kido, T.; Hongawa, K.; Ohuyama, K.; Nagai, K. Appl. Plws. Leth. $1994,64,815$.

8. Chao, C. I.; Chen, S. A. Appl. Phys. Lett. 1998, 73, 426.

9. Deshpande, R. S.: Bulovic, V:; Fonest, S. R. Appl Phys Lett. 1999, 75,888

10. Xie, Z. Y. et al. Appl. Plys. Len. 1999, 74,641

11. Kepler, R. G.; Beeson, P. M. Jacobs, S. T.; Anderson, R. A.; Sinclair, M. B. Appl Phus Letr. 1995, 66, 3618 .

12. Naka, S.; Okada, H.; Onnagawa, H.; Yamaguchi, Y.; Tsutsui, T. Synthetic Metals 2000, 111,331 .

13. Tang, C. W.: VanSlyke, S. A. Appl Phys Lett. 1987, 51, 913.

14. AziZ, H.; Popovic, Z. D.; Hu, N. X.; Hor, A. M.; Xu, G. Science $1999,283,1900$ 